\title{
Inonotus rickii (Agaricomycetes, Hymenochaetaceae) in Brazilian Cerrado: Expanding Its Geographic Distribution and Host List
}

\author{
Lucas Leonardo-Silva ${ }^{1 *}$, Ahmed M. Abdel-Azeem² and Solange Xavier-Santos' ${ }^{1}$ \\ ' Basic, Applied and Scientific Dissemination Micology Laboratory (FungiLab), Goiás State University, Anápolis, Brazil, \\ ${ }^{2}$ Department of Botany and Microbiology, Faculty of Science, Suez Canal University, Ismailia, Egypt
}

OPEN ACCESS

Edited by:

Dhanushka Nadeeshan

Wanasinghe,

Key Laboratory for Plant Diversity and Biogeography of East Asia, Kunming Institute of Botany, Chinese Academy of Sciences, China

Reviewed by: Zheng Wang, Yale University, United States Yu Pei Tan,

Department of Agriculture and Fisheries, Queensland

Government, Australia

*Correspondence:

Lucas Leonardo-Silva lucasleonardo@outlook.com;

lucasleo.bio@gmail.com

Specialty section: This article was submitted to

Evolutionary and Genomic Microbiology,

a section of the journal

Frontiers in Microbiology

Received: 30 December 2020

Accepted: 15 February 2021

Published: 09 March 2021

Citation:

Leonardo-Silva L

Abdel-Azeem AM and Xavier-Santos S (2021) Inonotus ricki

(Agaricomycetes,

Hymenochaetaceae) in Brazilian Cerrado: Expanding Its Geographic

Distribution and Host List.

Front. Microbiol. 12:647920. doi: 10.3389/fmicb.2021.647920
Inonotus rickii (Pat.) Reid (Agaricomycetes: Hymenochaetaceae) is a poroid fungus characterized by the expressive production of chlamydospores, in vivo and in vitro, especially during its anamorphic stage. The species plays important ecological roles, standing out as a phytopathogen, affecting several species of ornamental and wild trees, mainly in tropical and subtropical regions. The infected trees develop canker and white rot of the wood, showing symptoms of reduced vegetative vigor and decline of leaves and branches which causes death in some cases. The first record of I. rickii for the Cerrado biome (Brazilian Savanna) and the first record as causal agent of canker in Schinus molle L. in Brazil is reported here. In addition, we present a checklist of its worldwide geographical distribution and known hosts, from an extensive bibliographic search in Google Scholar, SciELO, Scopus, and Web of Science databases. The species is widespread in tropical and subtropical zones; common in the American continent, especially in Central and South America and the Mediterranean region, and rare in temperate zones. We found specimens growing in both living and dead hosts, totalizing 70 species of hosts, distributed in 43 genera and 22 families. Of these, Acer negundo L. (10.5\%), Celtis australis L. (6.5\%), and Platanus acerifolia (Aiton) Willd. (4.8\%), and the Fabaceae $(30 \%)$, Fagaceae $(10 \%)$, and Sapindaceae $(8.6 \%)$ families were the most frequent. We present morphological descriptions and illustrations, as well as the growth characteristics in culture medium. Our study expands the known geographical distribution of I. rickii, including the Cerrado biome, as well as its structural, physiological characteristics, and its hosts.

Keywords: Brazilian Savanna, Canker, decay, phytopathogen, poroid fungi

\section{INTRODUCTION}

Inonotus rickii (Pat.) Reid is a poroid fungus that belongs to the family Hymenochaetaceae, class Agaricomycetes. It was described in South America in 1896 as Ptychogaster cubensis Pat., from a review of specimens from Cuba by Patouillard (1896). However, in 1908, the author observed that the species should be the anamorphic stage of a poroid fungus collected by Ricki in Brazil, recognized as Xanthochrous rickii Pat. (Patouillard, 1908); later the nomenclature was changed to Polyporus rickii (Pat.) Sacc. and Trotter (Saccardo and Trotter, 1912). In 1957, the species was recognized by Reid as I. rickii (Reid, 1957), currently accepted nomenclature, and its anamorphic stage as $P$. cubensis. 
The relationship between the two life stages of the species has been confirmed by experimental studies carried by Davidson et al. (1942) and Stalpers (2000) and by molecular analysis of specimens collected from different geographical origins (Gottlieb et al., 2002; De Simone et al., 2011). I. rickii has a wide distribution in tropical, subtropical, and Mediterranean zones (Mazza et al., 2008; Ramos et al., 2008), where it is frequently found in trees in urban environments, mainly in its anamorphic stage (Mazza et al., 2008), which is considered a potential phytopathogen.

As a pathogen of woody plants, I. rickii infects branches and stems, causing canker and wood decay (Mazza et al., 2008; Annesi et al., 2010; De Simone et al., 2011). Adhesion and colonization of the substrate occurs due to the degradation of wood components, especially cellulose, hemicellulose and lignin, due to taxon enzymatic activity (Robles et al., 2014). When parasitizing the host, I. rickii can reach the heartwood, sapwood, and cambium, as well as provoke deep lesions resulting from the death of the bark tissues, characterizing the canker (Ramos et al., 2008; Annesi et al., 2010). Infected trees may show reduced vegetative growth, crown and branches decline and sparse foliage, which leads, in some cases, to death (Mazza et al., 2008; Ramos et al., 2008; Annesi et al., 2010).

The knowledge of this species-host began with reports describing the presence of a powdery mass of abundant ferruginous chlamydospores, which characterizes the anamorphic stage of the taxon in different plant hosts, but without mentioning its pathogenicity (Davidson et al., 1942). However, in the beginning of 2000, the diseases symptoms and pathological aspects became more evident in Europe. In Italy, the species was recorded in public gardens and wooded boulevards on Acer negundo L. and Albizia julibrissin Durazz. (Mazza et al., 2008; Annesi et al., 2010), and in Portugal, on Celtis australis L. (Ramos et al., 2008) causing serious damage or death of tree. Other reports in Chile (Sepúlveda et al., 2016), China (Cui et al., 2014), and Egypt (Shehata and Abdel-Wahab, 2013) also presented a similar situation regarding Schinus molle L., Acacia richii, A. Gray, Citrus spp. and Vitis spp. respectively. Based on the morphology of the basidiome and species of the host plants, the fungus called by different popular names e.g., "mapúa cheap," which means "mascarilla de la mapúa," in the Wayuu indigenous community of Colombia (Villalobos et al., 2017), or "Florcita de espinillo," "Florcita de molle," "Florcita de palo," "Hongo de espinillo," "Hongo de molle," and "Hongo de palo," in La Paz, Córboda, Argentina (Flamini et al., 2015).

In Brazil, information on $I$. rickii is mystery or fragmented and restricted to the holotype with unknown localities (Patouillard, 1908) and to occurrence records in the Caatinga biomes, on Spondias sp. (Umbuzeiro) (Drechsler-Santos et al., 2013; Maia et al., 2015); Atlantic Forest and Pampa without host identification (Campos-Santana and Loguercio-Leite, 2010; Maia et al., 2015). Here we report for the first time $I$. rickii in the Cerrado biome, which constitutes the first record of this species causing canker disease in $S$. molle. In addition, we present detailed morphological descriptions of both anamorphic and teleomorphic stages supported with illustrations, and updated checklist of its worldwide geographical distribution and known hosts up till now.

\section{MATERIALS AND METHODS}

\section{Study Area}

Samples were collected during the period from 2001 to 2020 in three conservation units of the Cerrado biome: the Estação

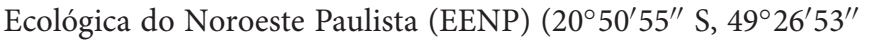
W), located between the municipalities of São José do Rio Preto and Mirassol, São Paulo; the Floresta Nacional (FLONA) de Silvânia $\left(16^{\circ} 38^{\prime} 30^{\prime \prime} \mathrm{S}, 48^{\circ} 39^{\prime} 02^{\prime \prime} \mathrm{W}\right)$, located in the municipality of Silvânia, Goiás; the Parque Estadual Altamiro de Moura Pacheco (PEAMP) $\left(16^{\circ} 33^{\prime} 12^{\prime \prime} \mathrm{S}, 49^{\circ} 8^{\prime} 50^{\prime \prime} \mathrm{W}\right)$, inserted in the municipalities of Goianápolis, Goiânia and Nerópolis, Goiás, and urban areas $\left(16^{\circ} 19^{\prime} 38^{\prime \prime} \mathrm{S}, 48^{\circ} 57^{\prime} 11^{\prime \prime} \mathrm{W}\right)$ in the municipality of Anápolis, Goiás, which are surrounded by several Cerrado fragments (Figure 1).

The Brazilian Cerrado is considered the richest savanna in the world and is the dominant biome in the Central Plateau of Brazil which covers part of the North, Northeast, South, and Southeast regions, representing approximately $25 \%$ of the country's territory. The Cerrado characterized by a rainy tropical climate, with high temperatures in summer and dry winter, this biome is known for its diverse landscapes represented by a vegetation mosaic, which includes forest, savanna, and grassland formations, where trees, shrubs, and undergrowth predominate, respectively (Klink and Machado, 2005; Ribeiro and Walter, 2008; WWF, 2015). Despite its great diversity, only $61 \%$ of the original Cerrado vegetation remains preserved (Sano et al., 2010). Sampling of our target species occurred in mesophilic forest (semideciduous dry forest) in which there is a predominance of tree species and canopy formation, occurring in interfluves and rich soil presented different levels of deciduous vegetation in the dry season (Ribeiro and Walter, 2008).

\section{Morphological Characterization}

Collected samples were dried at $40^{\circ} \mathrm{C}$ in oven and deposited in the Herbarium of the Universidade Estadual de Goiás (HUEG) and the Universidade Estadual Paulista (SJRP). Micro and macrophenotypic identification of basidiomata were carried out according to the relevant identification keys. For macroscopic characterization, the shape, consistency, color, dimension, and number of pores per $\mathrm{mm}$ of basidiomata were considered. For the description of the microscopic criteria, cross sections through the basidiomata in distilled water and $3 \% \mathrm{KOH}$ were microscopically examined. Hyphal structure, hymenial setae, setal hyphae, basidia, basidiospores, and chlamydospores and their ornaments were examined according to Ryvarden (2004, 2005) and Ramos et al. (2008). Melzer reagent was used to test the amyloid reaction of the microstructures (Kirk et al., 2008). All microscopic criteria were observed by Olympus CX31 optical microscope $(1000 \times)$ magnification, and the measurements were performed using the Piximètre software version 5.10 R 1541 (Henriot and Cheype, 2017). Abbreviations used for measurements of basidiospores and chlamydospores are: $Q=$ quotient between length and width, $\mathrm{Qm}=$ medium value of $Q$ and, $N=$ number of measured structures. All 

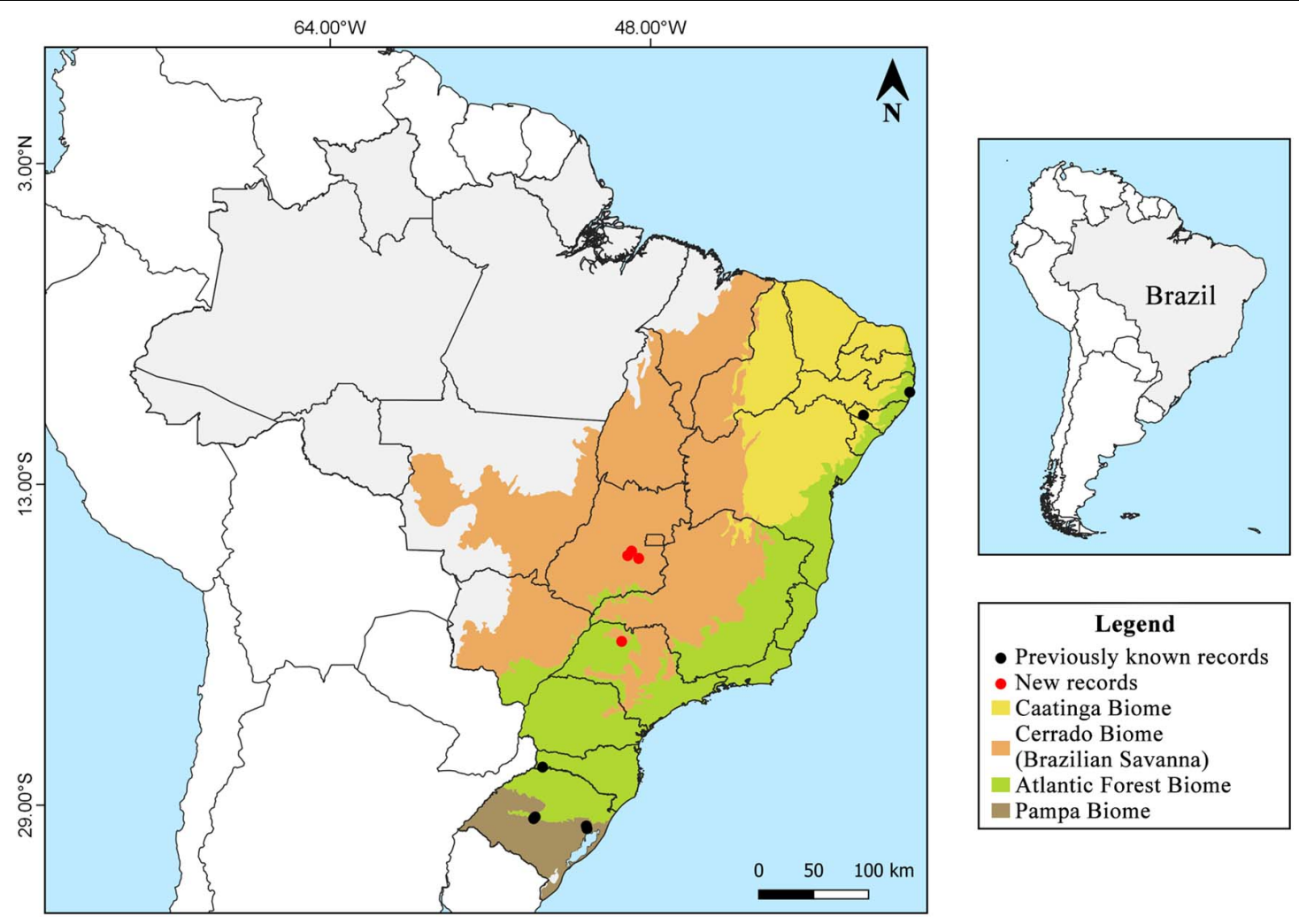

- Previously known records

- New records

Caatinga Biome

Cerrado Biome

(Brazilian Savanna)

Atlantic Forest Biome

Pampa Biome

FIGURE 1 | Study area and geographical distribution of Inonotus rickii in Brazil.

photographs and measurements were made using 3\% $\mathrm{KOH}$ as mounting medium. The color indication for all evaluated characters was based on Kornerup and Wanscher color cards (Kornerup and Wansher, 1978).

Fresh collected samples were plated out on potato dextrose agar (PDA) supplemented with $0.025 \mathrm{~g} / \mathrm{ml}^{-1}$ of chloramphenicol as bactericidal and incubated in a BOD incubator at $25^{\circ} \mathrm{C}$. Recovered colonies were characterized based on macro (shape, color, texture, and presence of exudates) and micromorphological criteria (hyphae, setal hyphae, and chlamydospores) according to relevant identification keys. The cultures were preserved according to Castellani (1963) and deposited in the collection of fungal cultures of the Laboratório de Micologia Básica, Aplicada e Divulgação Científica (FungiLab) of the Universidade Estadual de Goiás, Brazil.

\section{Checklist of Geographical Distribution and Hosts}

We collected the data from an extensive bibliographic search of Google Scholar ${ }^{1}, \mathrm{SciELO}^{2}$, Scopus ${ }^{3}$, and Web of Science ${ }^{4}$ by using different keywords: "Inonotus rickii" OR "Polyporus rickii" OR "Ptychogaster cubensis" OR "Xanthochrous rickii." Our data

\footnotetext{
${ }^{1}$ scholar.google.com.br

${ }^{2}$ www.scielo.org

${ }^{3}$ www.scopus.com

${ }^{4}$ www.webofknowledge.com
}

included all published articles, books, chapters, and abstracts available for access. For the worldwide geographical distribution of the species, we consider the specific location described by the authors and the geographic coordinates; when not available, they were obtained using the Latlong coordinate system (Latitude and Longitude Finder, 2020). The geographic distribution map was constructed using the Quantum GIS software (QGIS Development Team, 2020) and the climatic classification was determined between the North and South zones of both hemispheres as: tropical between $23^{\circ} 27^{\prime}$, subtropical $23^{\circ} 27^{\prime}$ and $46^{\circ} 54^{\prime}$, temperate $46^{\circ} 54^{\prime}$ and $66^{\circ} 33^{\prime}$, and polar $66^{\circ} 33^{\prime}$ and $90^{\circ}$, respectively (Peel et al., 2007; Eccles et al., 2019).

For the host checklist, we considered the absolute frequency the number of times the species was reported and relative frequency the quotient between the absolute frequency and the total of citations. The system of nomenclature, hierarchical classification and the name authority of the plant species followed The plant list (The Plant List, 2020).

\section{RESULTS}

\section{Taxonomic Treatment}

Inonotus rickii (Pat.) D. A. Reid, Kew Bull. [12](2): 141 (1957). (Figures 2, 3).

$\equiv$ Xanthochrous rickii Pat., Bull. Soc. Mycol. France 24(1): 6 (1908). 

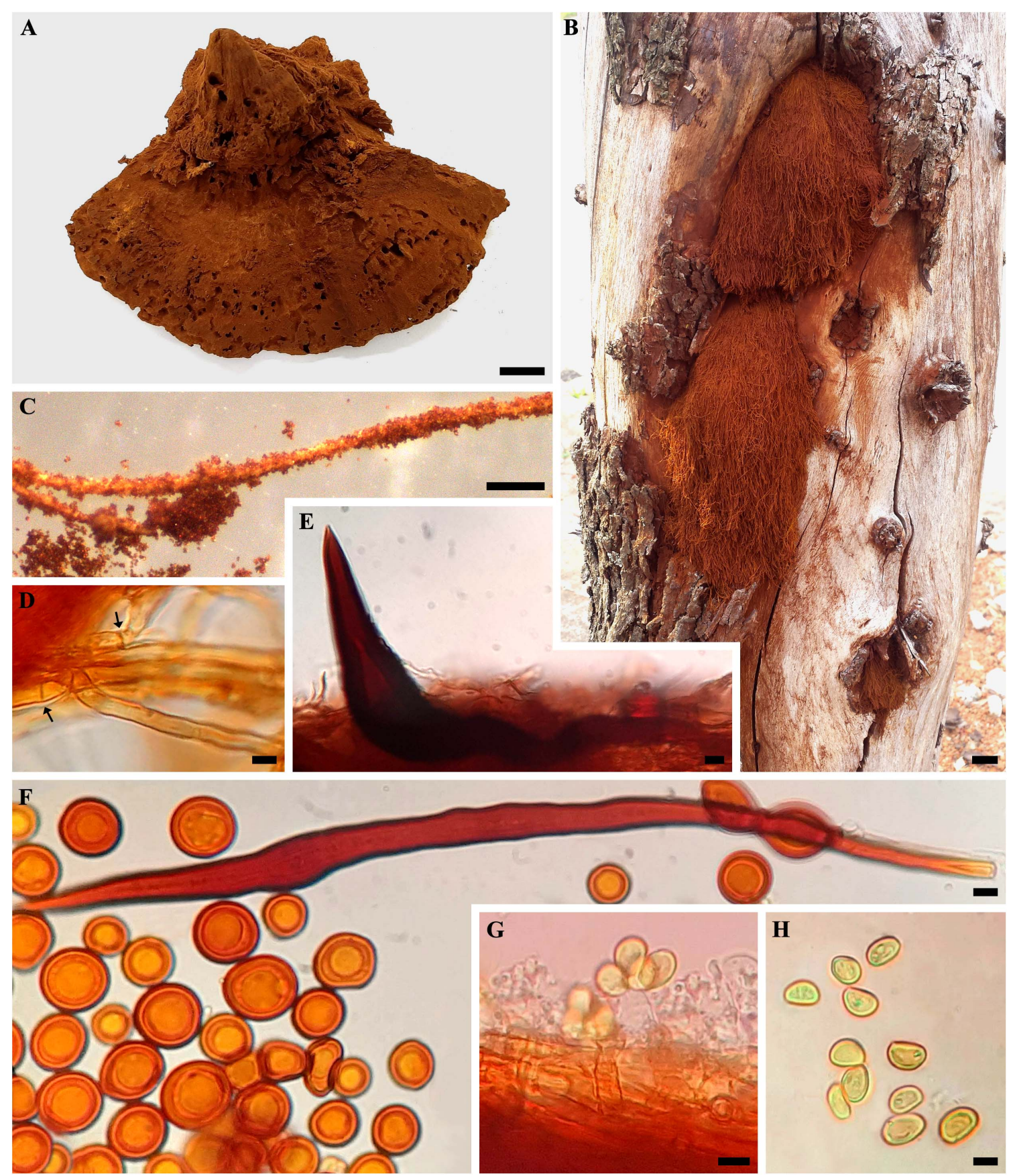

FIGURE 2 | Inonotus rickii. (A) Basidiome (HUEG 12067). (B) Anamorphic stage (HUEG 12062). (C) Chlamydospores attached to setal hyphae. (D) Generative hyphae, hyaline with simple septate (arrow). (E) Hymenial setae. (F) Setal hyphae and abundant chlamydospores. (G) Basidia hyaline, with projection of four sterigmatic structures and attached basidiospores. (H) Basidiospores. Bar $=1 \mathrm{~cm}(\mathbf{A}, \mathbf{B}) ; 5 \mathrm{~mm}$ (C); $5 \mu \mathrm{m}$ (D-H).

\section{Description}

Teleomorphic stage-basidiomata annual, $7.0-11 \times 6.0-7.8 \mathrm{~cm}$ in size, solitary or aggregated in the form of semicircular shelves, sessile, pileate, and strongly attached to the substrate. Pileus applanate to ungulate, margin obtuse to undulate, soft and spongy consistency when fresh and firm and crumbly when dry. Pileal surface covered by a dense powdery layer formed by reddish brown chlamydospore (8D7). Pore surface also covered by chlamydospore, circular to angular pores, 2-4 pores per $\mathrm{mm}$, thin and lacerated. Thick context and positive $\mathrm{KOH}$ reaction. Anamorphic stage-semicircular or cushionshaped, soft and robust, velvety to the touch, reddish orange (8B8) when young and reddish brown (8E7) when mature, structured by a dense mass of chlamydospore attached to hyphae, 

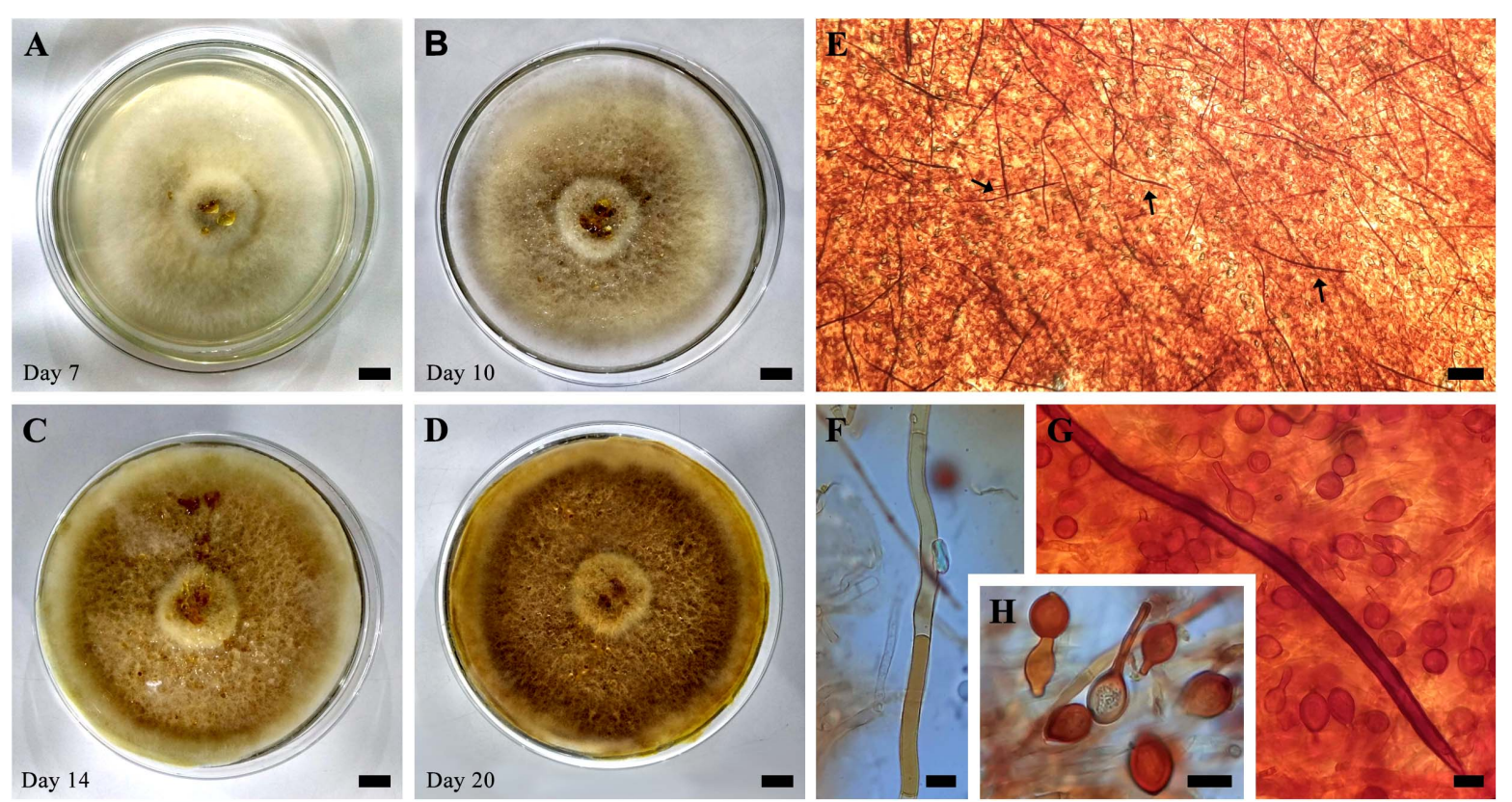

FIGURE 3 | (A-D) Evolution of growth in cultivation (SXS 37) in PDA at $25^{\circ} \mathrm{C}$ for 20 days. (E) Mycelial trama showing abundant setal hyphae (arrows). (F) Generative hyphae. (G) Setal hyphae and chlamydospores. (H) Chlamydospores. Bar = $1 \mathrm{~cm}$ (A-D); $100 \mu \mathrm{m}$ (E); $10 \mu \mathrm{m}$ (F-H).

forming a structure similar to capillitium, which facilitates dispersion by anemophilia.

Hyphal system monomitic, with generative hyphae, hyaline, septate, ranging from brownish yellow $(5 \mathrm{C} 7)$ to brownish orange (6C8), thin-walled to thick-walled, occasionally branched, 3.0$6.6 \mu \mathrm{m}$ in diam. Setal hyphae abundant in context, lanceolate, yellowish brown (5D5), thick-walled with a large lumen, with pointed apex, occasionally parallel to the hymenium, $8-20 \mu \mathrm{m}$ in diam. and 110-200 $\mu \mathrm{m}$ long. Hymenial setae abundant, ventricular to subulate, dark brown $(6 \mathrm{~F} 8), 14.3-63 \times 5.0-$ $18.4 \mu \mathrm{m}$, with thick-walled. Basidia hyaline, clavate to cylindric, $10 \times 6.5 \mu \mathrm{m}$, with projection of four sterigmatic structures. Basidiospores abundant, subglobose to ellipsoid, (5.4) 5.98.7 (9.5) $\times$ (3.6) 4.3-6.2 (6.5) $\mu \mathrm{m}[Q=$ (1) $1.2-1.6$ (2.1), $\mathrm{Qm}=1.4, N=50]$, golden yellow (5B7) to yellowish brown (5D5), dark brown (6F3), inamyloid, smooth with thick-walled. Chlamydospores abundant in the context, inamyloid, irregular, globose to subglobose, (7.4) 9.1-13.7 (15.5) $\times$ (6.9) 8.0-11.6 (13.9) $\mu \mathrm{m}[Q=1.0-1.3(1.9), \mathrm{Qm}=1.1, N=50]$, orange (6A8) to reddish orange (7A8), dark brown (6F3), smooth with thick-walled.

In culture presents velutinous to cottony mycelium, occupying the entire length of the petri dish $(90 \times 15 \mathrm{~mm})$ in 2 weeks of cultivation, yellowish white (2A2) when young, becoming grayish yellow (4B4) to dark yellow (4C8) when mature, production of exudates in the form of reddish orange droplets (8A8). Abundant production of chlamydospores of various shapes, occasionally attached to setal hyphae, also abundant and parallel in the hyphalic trama. The color and size characteristics of the cultures' microstructures are in accordance with those observed in the basidiomata.

\section{Worldwide Geographical Distribution}

Widespread in tropical and subtropical zones, considered then pantropical; common on the American continent, especially in Central and South America and the Mediterranean region, rare in temperate zones. There are records of the species in Argentina, Bahamas, Bermuda Islands, Brazil, Chile, China, Colombia, Costa Rica, Cuba, Egypt, France, Greece, Guadeloupe, Guinea, Haiti, India, Iran, Israel, Italy, Jamaica, Martinique, Mexico, Montenegro, Morocco, Myanmar, Pakistan, Paraguay, Peru, Philippines, Portugal, South Africa, Spain, Taiwan, United States, and Uruguay (Figure 4; Seaver and Waterston, 1946; Jaquenoud, 1985; Kotlaba and Pouzar, 1994; Chang and Fu, 1998; Stalpers, 2000; Gottlieb et al., 2002; Melo et al., 2002; Ryvarden, 2005; Martínez, 2006; Mata et al., 2007; Ghobad-Nejhad and Kotiranta, 2008; Annesi et al., 2010; Campos-Santana and Loguercio-Leite, 2010; Tura et al., 2010; De Simone et al., 2011; Ouabbou et al., 2012; Shehata and Abdel-Wahab, 2013; Valenzuela et al., 2013; Cui et al., 2014; García et al., 2014a; Robles et al., 2015a; Sepúlveda et al., 2016; Villalobos et al., 2017; Maubet, 2020; Tchoumi et al., 2020). Table 1 show detailed description of the species' worldwide geographic distribution, which contains the exact sampling location of each specimen described in the literature data found.

\section{Geographical Distribution in Brazil}

There are records in the Atlantic Forest areas in Pernambuco, Rio Grande do Sul and Santa Catarina, Caatinga areas in Alagoas and Bahia (record not shown in Figure 1, as the specific location was not found), Pampa areas in Rio Grande do Sul, and Cerrado areas (present study) in Goiás and São Paulo (Figure 1; CamposSantana and Loguercio-Leite, 2010; Maia et al., 2015). 


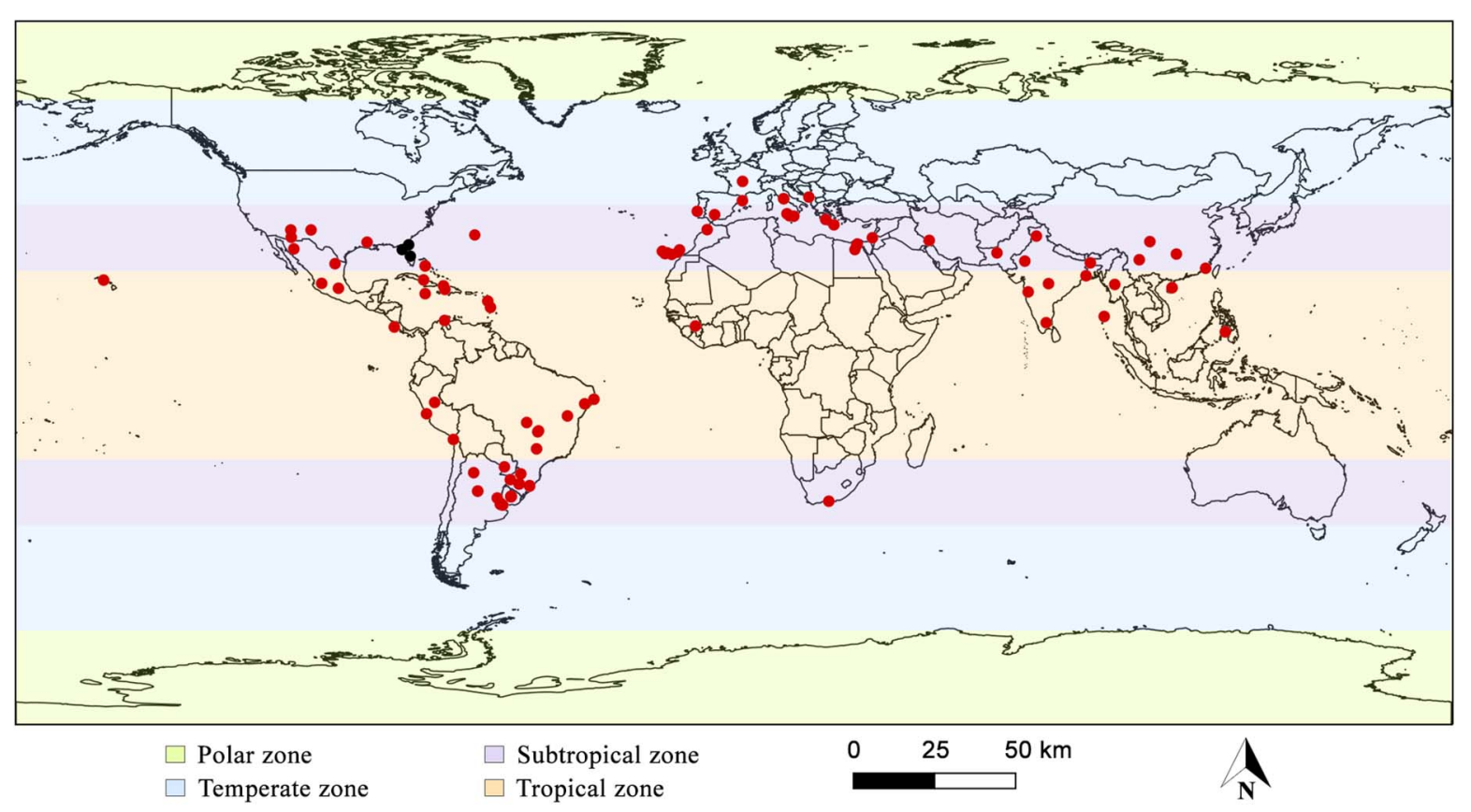

FIGURE 4 | Global geographical distribution of Inonotus rickii. Black dots highlight samples that may belong to another species, according to De Simone et al. (2011) and Cui et al. (2014).

\section{Habitat and Substrate}

We collect our specimens in the mesophilic forest in both stages of life, on dead wood and an unknown living host, and in urban areas in the anamorphic stage on living and dead S. molle trees. In the data set listed in the present review, we found 70 plant species distributed in 43 genera and 22 families (Table 2). Of these hosts, A. negundo (representing $10.5 \%$ of the total species), C. australis $6.5 \%$ and Platanus acerifolia $4.8 \%$. On the family level Fabaceae came first by accounted 30\% out of all host families followed by Fagaceae (10\%) and Sapindaceae (8.6\%). In the checklist, the host species referred as sp. or spp., were accounted for as a single species. Table 2 presents a complete list of I. rickii hosts.

\section{Material Examined}

BRAZIL. São Paulo: São José do Rio Preto-Mirassol, Estação Ecológica do Noroeste Paulista, 13/XI/2001, Xavier-Santos, S. (SJRP 28714), teleomorphic stage, found growing on fragment of dead wood, unknown host, isolated in culture with voucher number SXS 37; Goiás: Silvânia, Floresta Nacional de Silvânia, 26/VI/2009, Xavier-Santos, S. (HUEG 13945), anamorphic stage, growing on living tree, unknown host; GoianápolisGoiânia-Nerópolis, Parque Estadual Altamiro de Moura Pacheco, 08/VIII/2014, Xavier-Santos, S. (HUEG 12067), teleomorphic stage, found growing on living tree, unknown host; Anápolis, urban area, 20/III/2011, Xavier-Santos, S. (HUEG 13944), anamorphic stage, growing on living tree, unknown host; Parque Ipiranga, 19/IX/2018, Leonardo-Silva, L. (HUEG 12062), anamorphic stage, growing on a dead ornamental tree of S. molle, isolated in culture with voucher number SXS 641; ibid, 05/XII/2018, Leonardo-Silva, L. (HUEG 11993), anamorphic stage, growing on living S. molle; ibid, 27/V/2019, Leonardo-Silva, L. (HUEG 12063), anamorphic stage, growing on dead S. molle; ibid, 09/V/2020, Leonardo-Silva, L. (HUEG 12994), anamorphic stage, growing on living S. molle; ibid, 09/V/2020, Leonardo-Silva, L. (HUEG 12996), anamorphic stage, growing on dead S. molle; ibid, 09/V/2020, Leonardo-Silva, L. (HUEG 12997), anamorphic stage, growing on living S. mole; ibid, 10/XI/2020, Leonardo-Silva, L. (HUEG 13946), anamorphic stage, growing on dead S. mole.

\section{Examined Reference Material}

BRAZIL. Rio Grande do Sul: Santa Maria, 11/I/1993, Gilberto Coelho (24-9) (ICN 097679); Alagoas: São José da Tapera, 17/VI/2008, Drechsler-Santos (6) (URM 80418), on living tree, unknown host; ibid, 17/VI/2008, Drechsler-Santos (21) (URM 80460), growing on living tree of Spondias sp. (Umbuzeiro); ibid, 17/VI/2008, Drechsler-Santos (18) (URM 80582), an unknown living tree.

\section{Comments}

The morphological characteristics observed in collected samples coincide with those described by many authors (Gottlieb et al., 2002; Melo et al., 2002; Ryvarden, 2005). Measurements of setal hyphae recorded by Ryvarden (2005) and CamposSantana and Loguercio-Leite (2010) were up to $250 \mu \mathrm{m}$ or more long. Although these measurements are similar to our samples, we recorded smaller hyphal setae ranged between 110 and $200 \mu \mathrm{m}$ in the examined materials and pure cultures. I. rickii is similar to Inonotus patouillardii (Rick) Imazeki in the field but differentiating itself by the abundant presence of chlamydospores. Our specimens were collected during both rainy 
TABLE 1 | Referenced literature for the global geographical distribution of Inonotus rickii.

\begin{tabular}{|c|c|c|}
\hline Country & Locality & References \\
\hline \multirow[t]{15}{*}{ Argentina } & $\begin{array}{l}\text { Acassuso, Buenos Aires } \\
\text { Province }\end{array}$ & Gottlieb et al., 2002 \\
\hline & $\begin{array}{l}\text { Argentina (location not } \\
\text { specified) }\end{array}$ & Intini, 1988 \\
\hline & Buenos Aires City & $\begin{array}{l}\text { Gottlieb et al., 2002; Mielnichuk } \\
\text { and Lopez, 2007; Robles et al., } \\
\text { 2011, 2012, 2015a,b }\end{array}$ \\
\hline & Buenos Aires Province & Gottlieb et al., 2002 \\
\hline & Córdoba, Córdoba Province & Urcelay et al., 2012 \\
\hline & $\begin{array}{l}\text { Gualeguay, Entre Ríos } \\
\text { Province }\end{array}$ & Gottlieb et al., 2002 \\
\hline & $\begin{array}{l}\text { La Plata, Buenos Aires } \\
\text { Province }\end{array}$ & $\begin{array}{l}\text { Gottlieb et al., 2002; Wright } \\
\text { and Alberto, 2006; Murace } \\
\text { et al., } 2019\end{array}$ \\
\hline & $\begin{array}{l}\text { Llavallol, Buenos Aires } \\
\text { Province }\end{array}$ & Gottlieb et al., 2002 \\
\hline & $\begin{array}{l}\text { Lomas de Zamora, Buenos } \\
\text { Aires Province }\end{array}$ & Gottlieb et al., 2002 \\
\hline & $\begin{array}{l}\text { Martínez, Buenos Aires } \\
\text { Province }\end{array}$ & Gottlieb et al., 2002 \\
\hline & $\begin{array}{l}\text { Rosario del Tala, Entre Ríos } \\
\text { Province }\end{array}$ & Gottlieb et al., 2002 \\
\hline & $\begin{array}{l}\text { Santo Tomé, Corrientes } \\
\text { Province }\end{array}$ & Gottlieb et al., 2002 \\
\hline & Tucumán Province & Gottlieb et al., 2002 \\
\hline & $\begin{array}{l}\text { Valle de Traslasierra, Córdoba } \\
\text { Province }\end{array}$ & Flamini et al., 2015 \\
\hline & Yacanto, Córdoba Province & Gottlieb et al., 2002 \\
\hline \multirow[t]{2}{*}{ Bahamas } & $\begin{array}{l}\text { Bahamas (location not } \\
\text { specified) }\end{array}$ & Intini, 1988; Ryvarden, 2005 \\
\hline & Nassau & Davidson et al., 1942 \\
\hline $\begin{array}{l}\text { Bermuda } \\
\text { Islands }\end{array}$ & $\begin{array}{l}\text { Bermuda Islands (location not } \\
\text { specified) }\end{array}$ & Seaver and Waterston, 1946 \\
\hline \multirow[t]{10}{*}{ Brazil } & Anápolis, Goiás & Present study \\
\hline & Bahia & $\begin{array}{l}\text { Davidson et al., 1942; Stalpers, } \\
2000\end{array}$ \\
\hline & Brazil (location not specified) & $\begin{array}{l}\text { Davidson et al., 1942; } \\
\text { Ryvarden, 1983; Intini, } 1988\end{array}$ \\
\hline & Goiânia, Goiás & Present study \\
\hline & Ipojuca, Pernambuco & Maia et al., 2015 \\
\hline & Mondaí, Santa Catarina & $\begin{array}{l}\text { Campos-Santana and } \\
\text { Loguercio-Leite, } 2010\end{array}$ \\
\hline & $\begin{array}{l}\text { Porto Alegre, Rio Grande do } \\
\text { Sul }\end{array}$ & $\begin{array}{l}\text { Campos-Santana and } \\
\text { Loguercio-Leite, } 2010\end{array}$ \\
\hline & $\begin{array}{l}\text { Santa Maria, Rio Grande do } \\
\text { Sul }\end{array}$ & $\begin{array}{l}\text { Campos-Santana and } \\
\text { Loguercio-Leite, } 2010\end{array}$ \\
\hline & São José da Tapera, Alagoas & Drechsler-Santos et al., 2013 \\
\hline & $\begin{array}{l}\text { São José do Rio } \\
\text { Preto-Mirassol, São Paulo }\end{array}$ & Present study \\
\hline Chile & Arica, Arica Province & Sepúlveda et al., 2016 \\
\hline \multirow[t]{3}{*}{ China } & Hainan Province & $\begin{array}{l}\text { Cui et al., 2009; Dai et al., } \\
\text { 2010; Yuan et al., } 2015\end{array}$ \\
\hline & Jianyang, Sichuan Province & Zheng et al., 2011 \\
\hline & Panzhihua, Sichuan Province & $\begin{array}{l}\text { Chen et al., 2014; Cui et al., } \\
2014\end{array}$ \\
\hline Colombia & Uribia, Guajira & Villalobos et al., 2017 \\
\hline
\end{tabular}

(Continued)
TABLE 1 | Continued

\begin{tabular}{|c|c|c|}
\hline Country & Locality & References \\
\hline Costa Rica & Puntarenas, Cóbano & Mata et al., 2007 \\
\hline Cuba & Cuba (location not specified) & $\begin{array}{l}\text { Murrill, 1919; Intini, 1988; } \\
\text { Stalpers, } 2000\end{array}$ \\
\hline \multirow[t]{3}{*}{ Egypt } & Beni Suef Province & $\begin{array}{l}\text { Shehata and Abdel-Wahab, } \\
2013\end{array}$ \\
\hline & Giza Province & $\begin{array}{l}\text { Shehata and Abdel-Wahab, } \\
2013\end{array}$ \\
\hline & Qalubyia Province & $\begin{array}{l}\text { Shehata and Abdel-Wahab, } \\
2013\end{array}$ \\
\hline France & France (location not specified) & Ryvarden, 2005 \\
\hline \multirow[t]{2}{*}{ Greece } & Crete, Iráklion & Kotlaba and Pouzar, 1994 \\
\hline & Monemvasia, Laconia & De Simone et al., 2011 \\
\hline Guadeloupe & $\begin{array}{l}\text { Guadeloupe (location not } \\
\text { specified) }\end{array}$ & Jaquenoud, 1985; Intini, 1988 \\
\hline Guinea & $\begin{array}{l}\text { Guinea (location not } \\
\text { specified) }\end{array}$ & Jaquenoud, 1985; Intini, 1988 \\
\hline \multirow[t]{2}{*}{ Haiti } & Haiti (location not specified) & $\begin{array}{l}\text { Davidson et al., 1942; Intini, } \\
1988\end{array}$ \\
\hline & Tortuga Island & Davidson et al., 1942 \\
\hline \multirow[t]{7}{*}{ India } & $\begin{array}{l}\text { Andaman and Nicobar } \\
\text { Islands, Manjari }\end{array}$ & Sharma and Mishra, 2015 \\
\hline & Calcutta, West Bengal & Davidson et al., 1942 \\
\hline & Dindigul, Tamil Nadu & Mowna Sundari et al., 2018 \\
\hline & India (location not specified) & Intini, 1988; Ryvarden, 2005 \\
\hline & Jodhpur, Rajasthan & Singh et al., 2013 \\
\hline & Pune, Maharashtra & Jagtap et al., 2018 \\
\hline & $\begin{array}{l}\text { Western and Eastern } \\
\text { Himalaya }\end{array}$ & Sharma and Mishra, 2015 \\
\hline Iran & Khuzestan Province & $\begin{array}{l}\text { Ghobad-Nejhad and Kotiranta, } \\
2008\end{array}$ \\
\hline Israel & Tel Aviv & Ţura et al., 2010 \\
\hline \multirow[t]{4}{*}{ Italy } & Catania, Sicily & $\begin{array}{l}\text { Intini, 1988; Annesi et al., 2005, } \\
\text { 2010; De Simone et al., } 2011\end{array}$ \\
\hline & Palermo, Sicily & $\begin{array}{l}\text { Jaquenoud, 1985; Venturella } \\
\text { and Raimondo, 2004; Annesi } \\
\text { et al., 2005, 2010; Venturella } \\
\text { et al., 2006; De Simone et al., } \\
2011\end{array}$ \\
\hline & Roma & $\begin{array}{l}\text { Annesi et al., 2003, 2010, } \\
\text { 2005; Mazza et al., 2008; De } \\
\text { Simone et al., } 2011\end{array}$ \\
\hline & Sicily & Ryvarden, 2005 \\
\hline Jamaica & $\begin{array}{l}\text { Jamaica (location not } \\
\text { specified) }\end{array}$ & Jaquenoud, 1985; Intini, 1988 \\
\hline Martinique & Fort-de-France & $\begin{array}{l}\text { David and Rajchenberg, 1985; } \\
\text { Jaquenoud, 1985; Intini, } 1988\end{array}$ \\
\hline \multirow[t]{5}{*}{ Mexico } & Jalisco & Valenzuela et al., 2013 \\
\hline & Mexico City & Valenzuela et al., 2013 \\
\hline & Nuevo León & Valenzuela et al., 2013 \\
\hline & Sonora & $\begin{array}{l}\text { Esqueda et al., 2010; } \\
\text { Valenzuela et al., } 2013\end{array}$ \\
\hline & $\begin{array}{l}\text { Sonoran Desert, Nacapule } \\
\text { Canyon }\end{array}$ & Raymundo et al., 2013 \\
\hline Montenegro & Budva & Kotlaba and Pouzar, 1994 \\
\hline \multirow[t]{2}{*}{ Morocco } & Forest of Mamora & Ouabbou et al., 2012 \\
\hline & $\begin{array}{l}\text { Morocco (location not } \\
\text { specified) }\end{array}$ & Intini, 1988 \\
\hline
\end{tabular}

(Continued) 
TABLE 1 | Continued

\begin{tabular}{|c|c|c|}
\hline Country & Locality & References \\
\hline & Lima & Davidson et al., 1942 \\
\hline Myanmar & $\begin{array}{l}\text { Myanmar (location not } \\
\text { specified) }\end{array}$ & Jaquenoud, 1985; Intini, 1988 \\
\hline Pakistan & $\begin{array}{l}\text { Pakistan (location not } \\
\text { specified) }\end{array}$ & Jaquenoud, 1985; Intini, 1988 \\
\hline Paraguay & San Lorenzo & Maubet, 2020 \\
\hline Peru & Peru (location not specified) & Intini, 1988; Ryvarden, 2005 \\
\hline Philippines & Mindanao & Gottlieb et al., 2002 \\
\hline Portugal & Lisboa & $\begin{array}{l}\text { Melo et al., 2002; Ramos et al., } \\
2008\end{array}$ \\
\hline South Africa & $\begin{array}{l}\text { Knysna, Western Cape } \\
\text { Province }\end{array}$ & Tchoumi et al., 2017, 2020 \\
\hline \multirow[t]{10}{*}{ Spain } & Barcelona & De Simone et al., 2011 \\
\hline & Canary Islands, Rosa del Taro & García et al., 2014a \\
\hline & Córdoba & De Simone et al., 2011 \\
\hline & Gran Canaria, Canary Islands & Vergara et al., 2016 \\
\hline & La Gomera, Canary Islands & García et al., 2014b \\
\hline & La Palma, Canary Islands & García et al., 2014b \\
\hline & Lanzarote, Canary Islands & García et al., 2014b \\
\hline & Seville & $\begin{array}{l}\text { Intini, 2002a,b; Intini and Tello, } \\
2003\end{array}$ \\
\hline & Spain (location not specified) & Intini, 2002b \\
\hline & Tenerife, Canary Islands & García et al., 2014b \\
\hline Taiwan & Kinmen County & Chang and Fu, 1998 \\
\hline \multirow[t]{10}{*}{ United States } & Arizona & $\begin{array}{l}\text { Gillbertson et al., 1974; } \\
\text { Ryvarden, 2004, } 2005\end{array}$ \\
\hline & Florida & $\begin{array}{l}\text { Davidson et al., 1942; } \\
\text { Gillbertson and Ryvarden, 1986; } \\
\text { Intini, 1988; Barnard, 1993; } \\
\text { Ryvarden, 2004, } 2005\end{array}$ \\
\hline & Hawaii (location not specified) & Intini, 1988 \\
\hline & Hawthorne, Florida* & De Simone et al., 2011 \\
\hline & Jacksonville, Florida* & De Simone et al., 2011 \\
\hline & Levy County, Florida* & De Simone et al., 2011 \\
\hline & Louisiana & $\begin{array}{l}\text { Davidson et al., 1942; } \\
\text { Gilbertson and Ryvarden, 1986; } \\
\text { Intini, 1988; Ryvarden, 2004, } \\
2005\end{array}$ \\
\hline & New Mexico & Sanogo and Lujan, 2018 \\
\hline & Oahu, Hawaii & Davidson et al., 1942 \\
\hline & Tucson, Arizona & Gilbertson and Ryvarden, 1986 \\
\hline Uruguay & $\begin{array}{l}\text { Uruguay (location not } \\
\text { specified) }\end{array}$ & Martínez, 2006 \\
\hline
\end{tabular}

${ }^{*}$ Records of uncertain identification, according to De Simone et al. (2011) and Cui et al. (2014).

and drought periods which reflected the resistance to the climatic variations of the Cerrado.

\section{DISCUSSION}

Our study expands and shed the light on the geographic distribution of the species with special reference to the Cerrado biome and updated list of plant hosts. I. rickii is easily described by the massive production of chlamydospores in vitro and in vivo,
TABLE 2 | Checklist of hosts of Inonotus rickii reported in the literature.

\begin{tabular}{|c|c|c|c|}
\hline Family & Host species & AF & RF (\%) \\
\hline Adoxaceae & $\begin{array}{l}\text { Sambucus nigra L. (Kotlaba and Pouzar, } \\
\text { 1994; Annesi et al., 2003, 2005; De } \\
\text { Simone et al., 2011) }\end{array}$ & 4 & 3.2 \\
\hline \multirow[t]{5}{*}{ Anacardiaceae } & $\begin{array}{l}\text { Lithraea molleoides (Vell.) Engl. (Flamini } \\
\text { et al., 2015) }\end{array}$ & 1 & 0.8 \\
\hline & Pistacia atlantica Desf. (Vergara et al., 2016) & 1 & 0.8 \\
\hline & Schinus areira L. (Urcelay et al., 2012) & 1 & 0.8 \\
\hline & $\begin{array}{l}\text { Schinus molle L. (Intini, 1988, 2002b*; } \\
\text { Annesi et al., 2003; Intini and Tello, 2003**; } \\
\text { García et al., 2014a; Sepúlveda et al., 2016) }\end{array}$ & 5 & 4.0 \\
\hline & $\begin{array}{l}\text { Spondias sp. (Drechsler-Santos et al., } \\
\text { 2013) }\end{array}$ & 1 & 0.8 \\
\hline Altingiaceae & $\begin{array}{l}\text { Liquidambar styraciflua L. (Nakasone, } \\
\text { 1993) }\end{array}$ & 1 & 0.8 \\
\hline \multirow[t]{3}{*}{ Casuarinaceae } & $\begin{array}{l}\text { Casuarina cunninghamiana Miq. (Gottlieb } \\
\text { et al., 2002) }\end{array}$ & 1 & 0.8 \\
\hline & $\begin{array}{l}\text { Casuarina equisetifolia L. (Valenzuela et al., } \\
\text { 2013) }\end{array}$ & 1 & 0.8 \\
\hline & $\begin{array}{l}\text { Casuarina spp. (Gillbertson and Ryvarden, } \\
\text { 1986; Wright and Alberto, 2006) }\end{array}$ & 2 & 1.6 \\
\hline \multirow[t]{5}{*}{ Cannabaceae } & $\begin{array}{l}\text { Celtis australis L. (Kotlaba and Pouzar, } \\
\text { 1994; Gottlieb et al., 2002; Intini, 2002b**; } \\
\text { Melo et al., 2002; Annesi et al., 2003, 2015; } \\
\text { Intini and Tello, 2003**; Mazza et al., 2008; } \\
\text { Ramos et al., 2008) }\end{array}$ & 8 & 6.5 \\
\hline & $\begin{array}{l}\text { Celtis iguanaea (Jacq.) Sarg. as Celtis } \\
\text { ehrenbergiana (Klotzsch) Liebm. (Urcelay } \\
\text { et al., 2012) }\end{array}$ & 1 & 0.8 \\
\hline & Celtis spinosa Spreng. (Gottlieb et al., 2002) & 1 & 0.8 \\
\hline & $\begin{array}{l}\text { Celtis tala Gillies ex Planch. (Wright et al., } \\
\text { 1988, unpublished; Gottlieb et al., 2002) }\end{array}$ & 2 & 1.6 \\
\hline & Celtis sp. (Wright and Alberto, 2006) & 1 & 0.8 \\
\hline Euphorbiaceae & $\begin{array}{l}\text { Hevea brasiliensis (Willd. ex A. Juss.) Müll. } \\
\text { Arg. (Cui et al., 2009; Dai et al., 2010) }\end{array}$ & 2 & 1.6 \\
\hline \multirow[t]{14}{*}{ Fabaceae } & $\begin{array}{l}\text { Acacia caven (Molina) Molina (Flamini et al., } \\
\text { 2015) }\end{array}$ & 1 & 0.8 \\
\hline & Acacia koa A. Gray (Davidson et al., 1942) & 1 & 0.8 \\
\hline & $\begin{array}{l}\text { Acacia melanoxylon R. Br. (Gottlieb et al., } \\
\text { 2002) }\end{array}$ & 1 & 0.8 \\
\hline & $\begin{array}{l}\text { Acacia praecox Griseb. (Urcelay et al., } \\
\text { 2012) }\end{array}$ & 1 & 0.8 \\
\hline & Acacia richii A. Gray (Cui et al., 2014) & 1 & 0.8 \\
\hline & Acacia visco Griseb. (Urcelay et al., 2012) & 1 & 0.8 \\
\hline & $\begin{array}{l}\text { Albizia julibrissin Durazz. (Annesi et al., } \\
\text { 2007; Mazza et al., 2008) }\end{array}$ & 2 & 1.6 \\
\hline & $\begin{array}{l}\text { Albizia lebbeck (L.) Benth. (Ghobad-Nejhad } \\
\text { and Kotiranta, 2008) }\end{array}$ & 1 & 0.8 \\
\hline & Albizia sp. (Pieri and Rivoire, 1996) & 1 & 0.8 \\
\hline & $\begin{array}{l}\text { Cercidium sp. (Gilbertson and Ryvarden, } \\
\text { 1986) }\end{array}$ & 1 & 0.8 \\
\hline & $\begin{array}{l}\text { Delonix regia (Hook.) Raf. (Tura et al., 2009; } \\
\text { Țura et al., 2010; Jagtap et al., 2018; } \\
\text { Maubet, 2020) }\end{array}$ & 4 & 3.2 \\
\hline & Gleditsia sinensis Lam. (Zheng et al., 2011) & 1 & 0.8 \\
\hline & $\begin{array}{l}\text { Haematoxylon brasiletto H. Karst (Villalobos } \\
\text { et al., 2017) }\end{array}$ & 1 & 0.8 \\
\hline & $\begin{array}{l}\text { Parkinsonia aculeata L. (Gilbertson et al., } \\
\text { 1974; Jaquenoud, 1985) }\end{array}$ & 2 & 1.6 \\
\hline
\end{tabular}

(Continued) 
TABLE 2 | Continued

\begin{tabular}{|c|c|c|c|}
\hline Family & Host species & AF & RF (\%) \\
\hline & $\begin{array}{l}\text { Parkinsonia praecox (Ruiz and Pav.) } \\
\text { Hawkins (Villalobos et al., 2017) }\end{array}$ & 1 & 0.8 \\
\hline & $\begin{array}{l}\text { Parkinsonia spp. (Jaquenoud, 1985, 1987; } \\
\text { Gilbertson and Ryvarden, 1986; Ryvarden } \\
\text { and Gilbertson, 1993; Annesi et al., 2003) }\end{array}$ & 5 & 4.0 \\
\hline & $\begin{array}{l}\text { Prosopis cineraria (L.) Druce (Singh et al., } \\
\text { 2013) }\end{array}$ & 1 & 0.8 \\
\hline & $\begin{array}{l}\text { Prosopis juliflora (Sw.) DC. (Singh et al., } \\
\text { 2013; Villalobos et al., 2017) }\end{array}$ & 2 & 1.6 \\
\hline & $\begin{array}{l}\text { Robinia pseudoacacia L. (Mazza et al., } \\
\text { 2008; De Simone et al., 2011; Annesi et al., } \\
\text { 2015) }\end{array}$ & 3 & 2.4 \\
\hline & $\begin{array}{l}\text { Styphnolobium japonicum (L.) Schott } \\
\text { (Urcelay et al., 2012) }\end{array}$ & 1 & 0.8 \\
\hline & $\begin{array}{l}\text { Tamarindus indica L. (Davidson et al., 1942; } \\
\text { David and Rajchenberg, 1985**; } \\
\text { Jaquenoud, } 1987^{\star \star} \text { ) }\end{array}$ & 2 & 1.6 \\
\hline \multirow[t]{7}{*}{ Fagaceae } & $\begin{array}{l}\text { Quercus laevis Walter as Quercus } \\
\text { catesbaei Michx. (Davidson et al., 1942) }\end{array}$ & 1 & 0.8 \\
\hline & $\begin{array}{l}\text { Quercus cerris L. (Annesi et al., 2005; } \\
\text { Fodor and Hâruta, 2016) }\end{array}$ & 2 & 1.6 \\
\hline & $\begin{array}{l}\text { Quercus laurifolia Michx. (Gilbertson and } \\
\text { Ryvarden, 1986) }\end{array}$ & 1 & 0.8 \\
\hline & Quercus nigra L. (Davidson et al., 1942) & 1 & 0.8 \\
\hline & Quercus phellos L. (Davidson et al., 1942) & 1 & 0.8 \\
\hline & $\begin{array}{l}\text { Quercus sp. (Gilbertson and Ryvarden, } \\
\text { 1986) }\end{array}$ & 1 & 0.8 \\
\hline & $\begin{array}{l}\text { Quercus geminata Small as Quercus } \\
\text { virginiana var. geminata (Small) Sarg. } \\
\text { (Davidson et al., 1942) }\end{array}$ & 1 & 0.8 \\
\hline Icacinaceae & $\begin{array}{l}\text { Apodytes dimidiata E. Mey. ex Arn. as } \\
\text { Apodytes dimidiata subsp. dimidiata } \\
\text { (Tchoumi et al., 2017) }\end{array}$ & 1 & 0.8 \\
\hline Juglandaceae & $\begin{array}{l}\text { Carya illinoinensis (Wangenh.) K. Koch } \\
\text { (Sanogo and Lujan, 2018) }\end{array}$ & 1 & 0.8 \\
\hline \multirow[t]{2}{*}{ Malvaceae } & Chorisia spp. (Urcelay et al., 2012) & 1 & 0.8 \\
\hline & Brachychiton sp. (Murace et al., 2019) & 1 & 0.8 \\
\hline \multirow[t]{2}{*}{ Moraceae } & Ficus carica L. (Michailides, 2003) & 1 & 0.8 \\
\hline & Morus spp. (Urcelay et al., 2012) & 1 & 0.8 \\
\hline \multirow[t]{2}{*}{ Myricaceae } & $\begin{array}{l}\text { Morella cerifera (L.) Small as Myrica cerifera } \\
\text { L. (Davidson et al., 1942; Seaver and } \\
\text { Waterston, 1946; Gilbertson and Ryvarden, } \\
\text { 1986; De Simone et al., 2011*) }\end{array}$ & 4 & 3.2 \\
\hline & Myrica sp. (Gilbertson and Ryvarden, 1986) & 1 & 0.8 \\
\hline \multirow[t]{2}{*}{ Oleaceae } & Fraxinus sp. (Valenzuela et al., 2013) & 1 & 0.8 \\
\hline & $\begin{array}{l}\text { Olea capensis subsp. macrocarpa (C. H. } \\
\text { Wright) I. Verd. (Tchoumi et al., } \\
2017^{\star \star} \text { Tchoumi et al., } 2020^{\star \star} \text { ) }\end{array}$ & 1 & 0.8 \\
\hline \multirow[t]{3}{*}{ Platanaceae } & $\begin{array}{l}\text { Platanus acerifolia (Aiton) Willd. (Mazza } \\
\text { et al., 2008; Robles et al., 2011, 2012, } \\
\text { 2015a,b; Annesi et al., 2015) }\end{array}$ & 6 & 4.8 \\
\hline & $\begin{array}{l}\text { Platanus } \times \text { hispanica Mill. ex Münchh as } \\
\text { Platanus } \times \text { hybrida Brot. (Intini, 2002b; } \\
\text { Intini and Tello, 2003) }\end{array}$ & 1 & 0.8 \\
\hline & $\begin{array}{l}\text { Platanus spp. (Gottlieb et al., 2002; Intini } \\
\text { and Tello, 2003; Wright and Alberto, 2006) }\end{array}$ & 3 & 2.4 \\
\hline Rhamnaceae & $\begin{array}{l}\text { Ziziphus spina-christi (L.) Desf. } \\
\text { (Ghobad-Nejhad and Kotiranta, 2008) }\end{array}$ & 1 & 0.8 \\
\hline
\end{tabular}

(Continued)
TABLE 2 | Continued

\begin{tabular}{|c|c|c|c|}
\hline Family & Host species & AF & RF (\%) \\
\hline Rutaceae & $\begin{array}{l}\text { Citrus spp. (Shehata and Abdel-Wahab, } \\
\text { 2013) }\end{array}$ & 1 & 0.8 \\
\hline Salicaceae & $\begin{array}{l}\text { Dovyalis caffra (Hook.f. \& Harv.) Sim as } \\
\text { Aberia caffra Hook.f. \& Harv. (Annesi et al., } \\
\text { 2005) }\end{array}$ & 1 & 0.8 \\
\hline \multirow[t]{6}{*}{ Sapindaceae } & $\begin{array}{l}\text { Acer negundo L. (Pegler, 1967; Wright } \\
\text { et al., 1988; unpublished; Gottlieb et al., } \\
\text { 2002; Intini, 2002a*ıIntini, 2002b**; Annesi } \\
\text { et al., 2003, 2010, 2005; Intini and Tello, } \\
\text { 2003**; Venturella and Raimondo, 2004; } \\
\text { Venturella et al., 2006; Wright and Alberto, } \\
\text { 2006; Mazza et al., 2008; De Simone et al., } \\
\text { 2011; Murace et al., 2019) }\end{array}$ & 13 & 10.5 \\
\hline & $\begin{array}{l}\text { Acer saccharinum L. (Gilbertson and } \\
\text { Ryvarden, 1986) }\end{array}$ & 1 & 0.8 \\
\hline & Acer sp. (Gottlieb et al., 2002) & 1 & 0.8 \\
\hline & $\begin{array}{l}\text { Koelreuteria paniculata Laxm. (Annesi et al., } \\
\text { 2007; Mazza et al., 2008) }\end{array}$ & 2 & 1.6 \\
\hline & Melicoccus bijugatus Jacq. (Pegler, 1967) & 1 & 0.8 \\
\hline & Sapindus saponaria L. (Melo et al., 2002) & 1 & 0.8 \\
\hline Ulmaceae & Ulmus sp. (Murace et al., 2019) & 1 & 0.8 \\
\hline \multirow[t]{2}{*}{ Verbenaceae } & $\begin{array}{l}\text { Aloysia citriodora Palau as Lippia citriodora } \\
\text { (Palau) Kunth (Gottlieb et al., 2002) }\end{array}$ & 1 & 0.8 \\
\hline & Lippia sp. (Wright and Alberto, 2006) & 1 & 0.8 \\
\hline Vitaceae & $\begin{array}{l}\text { Vitis spp. (Shehata and Abdel-Wahab, } \\
\text { 2013) }\end{array}$ & 1 & 0.8 \\
\hline
\end{tabular}

Absolute frequency $(A F)=$ number of times that species was cited $(n=125)$; relative frequency $(R F)=$ percentage of citations of the species in relation to the total of host species found.

${ }^{*}$ Records of uncertain identification, according to De Simone et al. (2011) and Cui et al. (2014).

${ }^{* *}$ Articles that present the same data.

mainly in the anamorphic stage, and the presence of setal hyphae in the context (Pegler, 1964; Melo et al., 2002; Ramos et al., 2008; Tura et al., 2009; Maubet, 2020). In all of our specimens we observe these characteristics. Furthermore, the taxonomic criteria observed in pure cultures are compatible with those described by Ramos et al. (2008) and Tura et al. (2009).

Inonotus rickii is considered one of the most nocive basidiomycete in urban trees (Ramos et al., 2008), parasitizing a large number of hosts, thus being able to form an effective biological corridor in the dissemination of the pathogen in this environment (Annesi et al., 2010). The infection of these trees has a great impact in some regions of the world, as ornamental trees when parasitized and with symptoms of the disease, lose their ornamental value and represent a great danger to other members of the population (Ramos et al., 2008).

In Europe, especially in Italy (Annesi et al., 2010) and Portugal (Ramos et al., 2008), it has been reported that canker caused in plant tissues has reduced the number of ornamental tree species in urban environments. In addition, in the Wayuu indigenous community in Colombia, it has been reported that the fungus has had an impact on local native vegetation, also parasitizing species of daily use, such as Parkinsonia praecox 


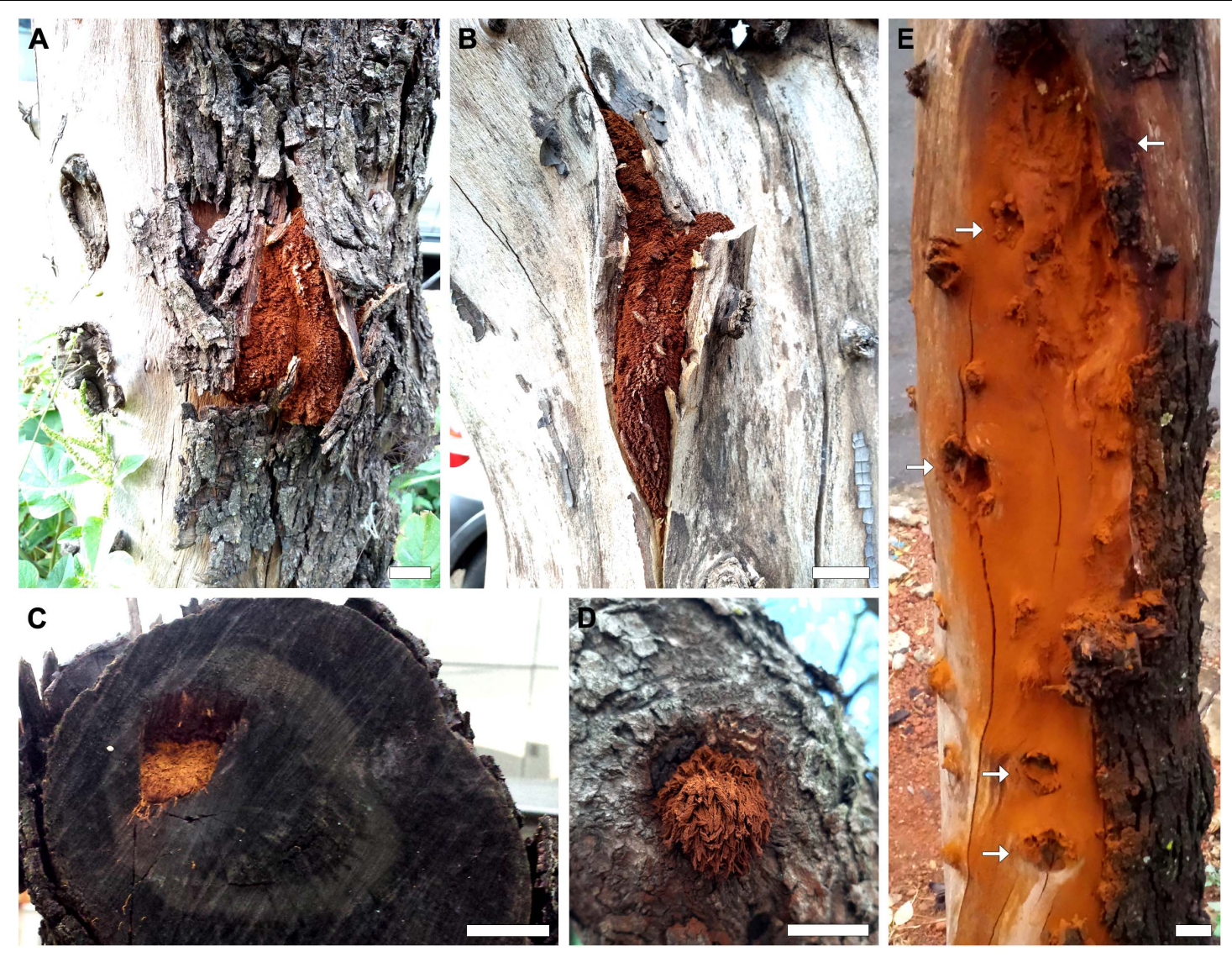

FIGURE 5 | Inonotus rickii in its anamorphic stage on Schinus molle. (A-C) Fruiting projecting between the bark, bark and heartwood of dead tree. (D) Initial projection of the fruiting body on living tree. (E) Powdery, rust-colored coating on the wood, which was formed by the deposition of chlamydospores when removing fruiting; it is also possible to observe the lesions (arrows) caused by the infection along the trunk. Bar $=2 \mathrm{~cm}$.

and Haematoxylon brasiletto, used for resin and paint extraction, respectively (Villalobos et al., 2017).

The most frequent host species of I. rickii are widely known as ornamental trees. However, some of the reported hosts are used as a food source (Barolo et al., 2014; Viveros Garciìa et al., 2018) or for extracting compounds for different applications (Sharma et al., 2018; Milena et al., 2019). This warns of the need to know the impact of $I$. rickii on species of local, regional and worldwide economics.

Our specimens were detected growing at the base of the stem and on main branches of living and dead trees, usually in the anamorphic stage. Only specimens SJRP 28714 and HUEG 12067 were sampled in their teleomorphic stage. Although the species produces reproductive structures in both stages of the life cycle, the teleomorphic phase occurs occasionally, with the anamorphic stage usually frequent (Mazza et al., 2008; Ramos et al., 2008).

The propagation of the species occurs mainly by the production and release of chlamydospores, which are more abundant in the anamorphic phase. At this stage, a structure sensitive to touch appears, which releases a large number of chlamydospores. On this occasion, in urban environments, dispersal is facilitated by tactile gardening activities in the maintenance of squares and parks, by anemophilia, by the contact of insects and other animals, including the flux of human traffic (Annesi et al., 2010). We observed that all specimens collected in an urban area (HUEG 11993, 12062, and 12063) were in the anamorphic stage and the dispersion of chlamydospores may have been facilitated by the activities mentioned before. Infection and development of the fungus occur when its spores (basidiospores or chlamydospores) are deposited on susceptible hosts, preferably due to the presence of dead wounds, scars, or fragments; these spores then germinate and promote the growth of the fungus within wood tissues (Phillips and Burdekin, 1989).

In the present study, we report for the first time occurrence of $I$. rickii on $S$. molle in Brazil. The fungus was found either as saprobic or as a pathogen, causing canker of the host plant. Belonging to the Anacardiaceae family, S. molle is an arboreal and perennial species, popularly known as pepper tree, pink pepper, American pepper, false pepper, aroeira salsa (Brazil) or aroeira mansa (Brazil). It is native to South America, but it was introduced and naturalized in several regions of the world, mainly as an ornamental in urban environments. Its great popularity as a cultivated plant is associated with its high tolerance to water and temperature availability, high growth rate and medicinal properties (Kramer, 1957; Goldstein and Coleman, 2004; Habte et al., 2020). 
Anamorphic samples infected S. molle (HUEG 11993, 12062, and 12063) were developed over the trunk and main branches of the living and dead host. We observed that the individuals growing in the dead host (HUEG 12062 and 12063) occupied a large extension of the wood between the bark and bark, projecting over the crevices of the stem and reaching to the heart (Figure 5). On the other hand, samples that grew on a living host (HUEG 11993), initial canker development was observed, possibly due to the time of maturity of the fungus that was in the early stages of development (Figure 5). The presence of I. rickii in this plant has already been reported in Portugal (Melo et al., 2002), Chile, and Spain (Intini and Tello, 2003; Sepúlveda et al., 2016).

Parallel to studies of phytopathological interest, I. rickii has also been the subject of studies to prospect for secondary metabolites and lignocellulolytic enzymes involved in attacking the host and degrading the constituents of wood (Xavier-Santos et al., 2004; Tura et al., 2009; Chen et al., 2014), aiming, above all, at an option sustainable in the bioremediation of recalcitrant compounds. The species is also associated with cultural events in the Wayuu indigenous community, Colombia, in which women apply a layer of chlamydospores to their face, previously treated with sheep tallow to protect themselves from solar radiation (Villalobos et al., 2017). Moreover, taxonomic and phylogenetic aspects are still performed, specially to understand its anamorphic stage.

Although molecular studies carried on different samples confirmed the relationship between anamorphic and teleomorphic phases of $I$. rickii. A preliminary phylogenetic analysis of samples (anamorphic and teleomorphic) collected from different geographical origins showed came from Florida, United States, were separated from those came from Asia, South America and Europe (De Simone et al., 2011; Cui et al., 2014). An interesting aspect to consider that all species from the Florida were collected in the anamorphic stage and the teleomorphic stage has never been recorded in North America. These results suggest the existence of two possible distinct species and are reinforced by morphophysiological, ecological data and climatic conditions (De Simone et al., 2011; Cui et al., 2014). However, studies that analyze isolates from North America and other regions, as well as the holotype of both stages of life, are necessary to confirm the distinction.

\section{CONCLUSION}

Our study expands the knowledge of geographical distribution of I. rickii, including the Cerrado biome, as well as of its structural,

\section{REFERENCES}

Annesi, T., Bressanin, D., Coppola, R., Mazza, G., and Motta, E. (2007). Osservazioni su Inonotus rickii in alberate cittadine. Micol. Ital. 36, 39-43.

Annesi, T., Calienno, L., Picchio, R., De Simone, D., and Lo Monaco, A. (2015). Degradation of some technological features in the wood of ornamental species caused by Inonotus rickii (Pat.) Reid. Drewno 58, 5-18. doi: 10.12841/wood. 1644-3985.136.01 physiological characteristics, and its hosts. Although common in tropical and subtropical regions, the specimens described here constitute the first documented records of the species for the Brazilian Cerrado and for the Midwest and Southeast regions, in addition to the first report on S. molle within Brazil. The checklist of hosts provided may assist in the development of practices for the control of the pathogen in urban areas with a high incidence of infections. Therefore, it should be mentioned here that, although the present study adds new data to information concerning $I$. rickii, this updated checklist must be considered as a provisional one always waiting for continuous supplementation.

\section{DATA AVAILABILITY STATEMENT}

The original contributions presented in the study are included in the article/supplementary material, further inquiries can be directed to the corresponding author/s.

\section{AUTHOR CONTRIBUTIONS}

LL-S and SX-S designed this study, collected and analyzed the samples, and wrote the manuscript text. LL-S prepared the figures and tables. AA-A reviewed and edited the manuscript. All authors have read and approved to the published version of the manuscript.

\section{FUNDING}

This study was financed in part by the Coordenação de Aperfeiçoamento de Pessoal de Nível Superior - Brasil (CAPES) Finance Code 001 (Convênio n 817164/2015 CAPES/PROAP).

\section{ACKNOWLEDGMENTS}

We are grateful to the Coordenação de Aperfeiçoamento de Pessoal de Nível Superior (CAPES), for the Ph.D. scholarship provided to the LL-S; to the administration of EENP, PEAMP, and Silvânia's FLONA for the support and authorization for collections in the area; to the To the ICN and URM herbaria, for lending material for comparative studies; to Hunter Lee Daniel, for reviewing the English language in the manuscript.

Annesi, T., Coppola, R., D’Amico, L., and Motta, E. (2005). First report of Aberia caffra and Quercus cerris as hosts of Inonotus rickii. Plant Dis. 89:107. doi: 10.1094/PD-89-0107C

Annesi, T., Coppola, R., and Motta, E. (2003). Decay and canker caused by Inonotus rickii spreading on more urban tree species. For. Pathol. 33, 405-412. doi: 10.1046/j.1437-4781.2003.00340.x

Annesi, T., D'Amico, L., Bressanin, D., Motta, E., and Mazza, G. (2010). Characterization of Italian isolates of Inonotus rickii. Phytopathol. Mediterr. 49, 301-308. doi: 10.14601/Phytopathol_Mediterr-8402 
Barnard, E. L. (1993). Inonotus rickii (Anamorph: Ptychogaster cubensis): a wooddecaying fungus of importance to Florida. Plant Pathol. Circ. 10-12.

Barolo, M. I., Ruiz Mostacero, N., and López, S. N. (2014). Ficus carica L. (Moraceae): an ancient source of food and health. Food Chem. 164, 119-127. doi: 10.1016/j.foodchem.2014.04.112

Campos-Santana, M., and Loguercio-Leite, C. (2010). Austro-American lignocellulolytic basidiomycetes (Agaricomycotina): new records. Mycotaxon 114, 377-392. doi: 10.5248/114.377

Castellani, A. (1963). Further researches on the long viability and growth of many pathogenic fungi and some bacteria in sterile distilled water. Mycopathol. Mycol. Appl. 20, 1-6. doi: 10.1007/BF02054872

Chang, T., and Fu, C.-S. (1998). Studies on eight species of polypores from Taiwan. Fungal Sci. 13, 75-83.

Chen, H.-P., Dong, W.-B., Feng, T., Yin, X., Li, Z.-H., Dong, Z.-J., et al. (2014). Four new sesquiterpenoids from fruiting bodies of the fungus Inonotus rickii. J. Asian Nat. Prod. Res. 16, 581-586. doi: 10.1080/10286020.2014.925451

Cui, B. K., Yu, C. J., and Li, H. J. (2009). Two species of Inonotus (Hymenochaetaceae) new to China. For. Res. 22, 784-787.

Cui, B. K., Zhao, C. L., Vlasák, J., and Dai, Y. C. (2014). A preliminary report on decay and canker of Acacia richii caused by Inonotus rickii in China. For. Pathol. 44, 82-84. doi: 10.1111/efp.12088

Dai, Y. C., D'Amico, L., Motta, E., and Annesi, T. (2010). First report of Inonotus rickii causing canker and decay on Hevea brasiliensis in China. Plant Pathol. 59, 806-806. doi: 10.1111/j.1365-3059.2009.02253.x

David, A., and Rajchenberg, M. (1985). Pore fungi from French Antilles and Guiana. Mycotaxon 22, 285-325.

Davidson, R. W., Campbell, W. A., and Weber, G. F. (1942). Ptychogaster cubensis, a wood-decaying fungus of southern oaks and waxmyrtle. Mycol. Soc. Am. 34, 142-153. doi: 10.1080/00275514.1942.12020885

De Simone, D., D'Amico, L., Bressanin, D., Motta, E., and Annesi, T. (2011). Molecular characterization of Inonotus rickii/Ptychogaster cubensis isolates from different geographic provenances. Mycol. Prog. 10, 301-306. doi: 10.1007/ s11557-010-0702-5

Drechsler-Santos, E. R., Gibertoni, T. B., Calvacanti, M. A., de, Q., Ryvarden, L., and Góes-Neto, A. (2013). "Basidiomycota polypores - orelhas de pau," in Guide to the Common Fungi of the Semiarid Region of Brazil, eds M. A. Neves, I. Baseia, E. R. Drechsler-Santos, and A. Góes-Neto (Florianópolis: Tecc Editora).

Eccles, R., Zhang, H., and Hamilton, D. (2019). A review of the effects of climate change on riverine flooding in subtropical and tropical regions. J. Water Clim. Chang. 10, 687-707. doi: 10.2166/wcc.2019.175

Esqueda, M., Coronado, M., Gutierrez, A., Valenzuela, R., Chacón, S., Gilbertson, R. L., et al. (2010). "Hongos," in Diversidad Biológica de Sonora, eds F. E. M. Freaner and T. R. Van Devender (Mexico: BMC Public Health), 496.

Flamini, M., Robledo, G. L., and Suárez, M. E. (2015). Nombres y clasificaciones de los hongos según los campesinos de La Paz (Valle de Traslasierra, Córdoba, Argentina). Bol. Soc. Argent. Bot. 50, 265-289. doi: 10.31055/1851.2372.v50.n3. 12518

Fodor, E., and Hâruta, O. (2016). Quercus Robur, Q. Cerris and Q. Petraea as hot spots of biodiversity. J. Plant Dev. 23, 187-210.

García, V. J. E., Inchaurtieta, E. B., Martín, L. C., López-Quintanilla, J. F., Rodríguez-Cabrera, C. C., and Vergara, J. I. V. (2014a). Dos nuevos hongos para la isla de Fuerteventura. Majorensis 10, 29-36.

García, V. J. E., Romero, D. B., Cabrera, C. C. R., and Godoy, S. J. (2014b). "Revisión y actualización de la biota fúngica de la isla de Fuerteventura," in Proceedings of the XXI Coloquio de Histo- ria Canario-Americana (2014), (Casa de colon).

Ghobad-Nejhad, M., and Kotiranta, H. (2008). The genus Inonotus sensu lato in Iran, with keys to Inocutis and Mensularia worldwide. Ann. Bot. Fenn. 45, 465-476. doi: 10.5735/085.045.0605

Gilbertson, R. L., Martin, K. J., and Lindsey, J. P. (1974). Annotated Check List and Host Index for Arizona Wood-Rotting Fungi. Tucson, AZ: University of Arizona.

Gilbertson, R. L., and Ryvarden, L. (1986). North American Polypores. Oslo: Fungiflora.

Goldstein, D. J., and Coleman, R. C. (2004). Schinus molle L. (Anacardiaceae) Chicha production in the Central Andes. Econ. Bot. 58, 523-529. doi: 10.1663/ 0013-0001(2004)058[0523:SMLACP]2.0.CO;2

Gottlieb, A. M., Wright, J. E., and Moncalvo, J. M. (2002). Inonotus s. 1. in Argentina - morphology, cultural characters and molecular analyses. Mycol. Prog. 1, 299-313. doi: 10.1007/s11557-006-0028-5
Habte, G., Nedi, T., and Assefa, S. (2020). Antimalarial activity of aqueous and $80 \%$ methanol crude seed extracts and solvent fractions of Schinus molle Linnaeus (Anacardiaceae) in Plasmodium berghei -Infected mice. J. Trop. Med. 2020:9473250. doi: 10.1155/2020/9473250

Henriot, A., and Cheype, J. L. (2017). Piximètre, la Measure Des Dimensions Sur Images. Available online at: http://www.piximetre.fr/ [Accessed November 13, 2020]

Intini, M. (1988). Contributo alla conoscenza dei funghi lignicoli italiani: Inonotus rickii (Pat.) Reid. Micol. Ital. 1, 49-53.

Intini, M. (2002a). First report of Inonotus rickii causing canker rot on boxelder in Europe. Plant Dis. 86, 922-922. doi: 10.1094/PDIS.2002.86.8.922C

Intini, M. (2002b). Inonotus rickii found in Spain. Micol. Veg. Mediterr. 17, 25-30.

Intini, M., and Tello, M. (2003). Comunicación, investigaciones sobre hongos xilófagos de arboles urbanos en Europa: primera cita de Inonotus rickii (Pat.) Reid en España. Bol. Sanid. Veg. Plagas 29, 277-279.

Jagtap, N., Ranadive, K., Jagtap, P., Chaudhari, N., Thopate, P., Jagtap, P., et al. (2018). Bioactive potentials of Inonotus rickii (Pat.) D. A. reid from Pune. Ajanta 7, 184-193.

Jaquenoud, M. (1985). Inonotus rickii, un polypore nouveau pour la flore européenne. Mycol. Helv. 1, 371-391.

Jaquenoud, M. (1987). Les cellules hymèniales d' Inonotus rickii trouvé en Europe. Mycol. Helv. 2, 209-215.

Kirk, P. M., Cannon, P. F., Minter, D. W., and Stalpers, J. A. (2008). Dictionary of the Fungi, 10th Edn. Wallingford: CABI.

Klink, C. A., and Machado, R. B. (2005). A conservação do Cerrado brasileiro. Megadiversidade 1, 147-155. doi: 10.1590/S0100-69912009000400001

Kornerup, A., and Wansher, J. H. (1978). Handbook of Colour, 3rd Edn. London: Eyre Methuen.

Kotlaba, F., and Pouzar, Z. (1994). Two new localities of Inonotus rickii in Europe. Czech Mycol. 47, 159-161. doi: 10.33585/cmy.47205

Kramer, F. L. (1957). The pepper tree, Schinus molle L. Econ. Bot. 11, 322-326. doi: 10.1007/BF02903811

Latitude and Longitude Finder (2020). Available online at: https://www.latlong.net/ [Accessed March 3, 2020].

Maia, L. C., De Carvalho Júnior, A. A., Cavalcanti, L. D. H., Gugliotta, A. D. M., Drechsler-Santos, E. R., Santiago, A. L. M. D. A., et al. (2015). Diversity of brazilian fungi. Rodriguesia 66, 1033-1045. doi: 10.1590/2175-7860201566407

Martínez, S. (2006). The genera Inocutis and Inonotus (Hymenochaetales) in Uruguay. Mycotaxon 96, 1-8.

Mata, M., Ruiz-Boyer, A., Carranza, J., and Ryvarden, L. (2007). Nuevos registros de hongos poliporoides (Basidiomycetes) para Costa Rica. Bol. Soc. Micol. Madr. 31, 123-130.

Maubet, Y. (2020). Hymenochaetaceae from Paraguay: revision of the family and new records. Curr. Res. Environ. Appl. Mycol. 10, 242-261. doi: 10.5943/cream/ $10 / 1 / 24$

Mazza, G., Moriondo, M., Motta, E., and Annesi, T. (2008). Monitoraggio fitopatologico di Inonotus rickii nella città di Roma e applicazioni GPS-GIS. Forest@ 5, 160-170. doi: 10.3832/efor0523-0050160

Melo, I., Ramos, P., and Caetano, M. F. F. (2002). First record of Inonotus rickii (Basidiomycetes, Hymenochaetaceae) in Portugal. Port. Acta Biol. 20, 265-269.

Michailides, T. J. (2003). "Diseases of Fig," in Diseases of Tropical Fruit Crops, ed. R. C. Ploetz (Wallingford: CABI), 527.

Mielnichuk, N., and Lopez, S. E. (2007). Interaction between Epicoccum purpurascens and xylophagous basidiomycetes on wood blocks. For. Pathol. 37, 236-242. doi: 10.1111/j.1439-0329.2007.00496.x

Milena, V., Tatjana, M., Gökhan, Z., Ivana, B., Aleksandra, C., Mohammad, M. F., et al. (2019). Advantages of contemporary extraction techniques for the extraction of bioactive constituents from black elderberry (Sambucus nigra L.) flowers. Ind. Crops Prod. 136, 93-101. doi: 10.1016/j.indcrop.2019.04.058

Mowna Sundari, T., Alwin Prem Anand, A., Jenifer, P., and Shenbagarathai, R. (2018). Bioprospection of Basidiomycetes and molecular phylogenetic analysis using internal transcribed spacer (ITS) and 5.8S rRNA gene sequence. Sci. Rep. 8:10720. doi: 10.1038/s41598-018-29046-w

Murace, M., Luna, M. L., Robledo, G., and Perelló, A. (2019). "Pudrición del leño en los árboles urbanos," in Problemáticas Sanitarias del Arbolado. Enfermedades presentes en La Plata. Especial referencia a las de origen fúngico, eds A. M. Aprea and M. A. Murace (Buenos Aires: Editorial de la Universidad de La Plata), 156. 
Murrill, W. A. (1919). Cuban polypores and agarics. Mycologia 11, 22-32. doi: 10.1080/00275514.1919.12016771

Nakasone, K. K. (1993). "Diversity of lignicolous Basidiomycetes in coarse woody debris," in Biodiversity and Coarse Woody Debris in Southern Forests, eds J. W. McMim, D. A. J. Crossley, and G. A. Athens (Asheville, NC: USDA Forest Service Southern Research Station), 35-39.

Ouabbou, A., El-assfouri, A., Ouazzani-Touhami, A., Benkirane, R., and Douira, A. (2012). Bibliographic catalog of the forest of Mamora (Morocco) fungal flora. J. Anim. Plant Sci. 15, 2200-2242.

Patouillard, N. (1896). Champignons nouveaux ou peu connus. Bull. Soc. Bot. Fr. $12,132-136$

Patouillard, N. (1908). Champignons nouveaux ou peu connus. Bull. Soc. Bot. Fr. 24, 1-12.

Peel, M. C., Finlayson, B. L., and McMahon, T. A. (2007). Updated world map of the Köppen-Geiger climate classification. Hydrol. Earth Syst. Sci. 11, 1633-1644. doi: 10.5194/hess-11-1633-2007

Pegler, D. N. (1964). A survey of the genus Inonotus (Polyporaceae). Trans. Br. Mycol. Soc. 47, 175-195. doi: 10.1016/S0007-1536(64)80051-6

Pegler, D. N. (1967). Notes on Indian Hymenochaetoïdeae. Kew Bull. 21, 39-49. doi: $10.2307 / 4108418$

Phillips, D. H., and Burdekin, D. A. (1989). Diseases of Forest and Ornamental Trees. London: MACMILLAN.

Pieri, M., and Rivoire, B. (1996). À propos de quelques polypores (Aphyllophoromycetideae) rares ou critiques récoltes récemment. I. Bull. Soc. Mycol. Fr. 112, 163-187.

QGIS Development Team (2020). QGIS Geographic Information System. Open Source Geospatial Foundation Project. Available online at: https://qgis.org/en/ site/ (accessed November 13, 2020).

Ramos, A. P., Caetano, M. F., and Melo, I. (2008). Inonotus rickii (Pat.) Reid: an important legnicolous Basidiomycete in urban trees. Rev. Ciênc. Agrár. 31, 159-167. doi: 10.19084/rca.15616

Raymundo, T., Valenzuela, R., Gutiérrez, A., Coronado, M. L., and Esqueda, M. (2013). Agaricomycetes xilófagos de la planicie central del desierto sonorense. Rev. Mex. Biodivers. 84, 417-424. doi: 10.7550/rmb.30828

Reid, D. A. (1957). New or interesting records of Australasian Basidiomycetes: III. Kew Bull. 12, 127-143. doi: 10.2307/4109115

Ribeiro, J. F., and Walter, B. M. T. (2008). "As principais fitofisionomias do bioma Cerrado," in Cerrado: Ecologia e flora, eds S. M. Sano, S. P. Almeida, and J. F. Ribeiro (Brasília, DF: Embrapa Informação Tecnológica), 152-212.

Robles, C. A., Carmarán, C. C., and Lopez, S. E. (2011). Screening of xylophagous fungi associated with Platanus acerifolia in urban landscapes: biodiversity and potential biodeterioration. Landsc. Urban Plan. 100, 129-135. doi: 10.1016/j. landurbplan.2010.12.003

Robles, C. A., Castro, M. A., and Castro, M. A. (2014). Wood decay by Inonotus rickii and Bjerkandera adusta: a micro- and ultra-structural approach. IAWA J. 35, 51-60. doi: 10.1163/22941932-00000047

Robles, C. A., Lopez, S. E., and Carmarán, C. C. (2015a). Basidiomicetes endofíticos de madera en Platanus acerifolia (Platanaceae) de Argentina: notas y estudios de cultivo. Bol. Soc. Argent. Bot. 50, 437-445. doi: 10.31055/1851.2372.v50.n4. 12907

Robles, C. A., Lopez, S. E., McCargo, P. D., and Carmarán, C. C. (2015b). Relationships between fungal endophytes and wood-rot fungi in wood of Platanus acerifolia in urban environments. Can. J. For. Res. 45, 929-936. doi: $10.1139 /$ cjfr-2014-0560

Robles, C., Carmarán, C., and Lopez, S. (2012). Molecular techniques based on ITS analysis: useful tools for the identification of wood-decay Basidiomycetes in urban trees? Kurtziana 37, 91-108.

Ryvarden, L. (1983). Type studies in the Polyporaceae 14. species described by N. Patouillard, either alone or with other mycologists. Harvard University, Cambridge, MA.

Ryvarden, L. (2004). Neotropical Polypores Part 1. Oslo: Fungiflora.

Ryvarden, L. (2005). The Genus Inonotus a Synopsis. Oslo: Fungiflora.

Ryvarden, L., and Gilbertson, R. L. (1993). European Polypores. Oslo: Fungiflora.

Saccardo, P. A., and Trotter, A. (1912). Sylloge fungorum omnium hucusque cognitorum. Bull. Soc. Bot. Fr. 21:270.

Sano, E. E., Rosa, R., Brito, J. L. S., and Ferreira, L. G. (2010). Land cover mapping of the tropical savanna region in Brazil. Environ. Monit. Assess. 166, 113-124. doi: 10.1007/s10661-009-0988-4
Sanogo, S., and Lujan, P. (2018). Rarity of a fungal pathogen and a parasitic flowering plant versus the commonness of a mycorrhizal fungus in Pecan Orchards in New Mexico. Plant Heal. Prog. 19, 207-211. doi: 10.1094/PHP-0518-0024-S

Seaver, F. J., and Waterston, J. M. (1946). Contributions to the Mycoflora of Bermuda-IV. Mycologia 38, 180-201. doi: 10.1080/00275514.1946.1202 4051

Sepúlveda, G., Huanca, W., Cárdenas, S., Belmonte, E., Torres, T., and Latorre, B. A. (2016). First report of Inonotus rickii causing decay and death in Schinus molle in Chile. Plant Dis. 100:2320. doi: 10.1094/PDIS-01-16-00 10-PDN

Sharma, J. R., and Mishra, D. (2015). A Synoptic mycoflora of wood-rotting fungi of Andaman. Nelumbo 57, 135-164. doi: 10.20324/nelumbo/v57/2015/8 7118

Sharma, S., Sharma, S., and Vig, A. P. (2018). Antigenotoxic potential of plant leaf extracts of Parkinsonia aculeata L. using Allium cepa assay. Plant Physiol. Biochem. 130, 314-323. doi: 10.1016/j.plaphy.2018.07.017

Shehata, A. S. F., and Abdel-Wahab, F. M. (2013). First record of Ptychogaster cubensis causing canker and decay on citrus and grape trees in Egypt. Egypt. J. Phytopathol. 41, 215-216. doi: 10.21608/ejp.2013.10 2006

Singh, S. K., Doshi, A., Pancholy, A., and Pathak, R. (2013). Biodiversity in wooddecay macro-fungi associated with declining arid zone trees of India as revealed by nuclear rDNA analysis. Eur. J. Plant Pathol. 136, 373-382. doi: 10.1007/ s10658-013-0172-0

Stalpers, J. A. (2000). The genus Ptychogaster. Karstenia 40, 167-180. doi: 10. 29203/ka.2000.368

Tchoumi, J. M. T., Coetzee, M. P. A., Rajchenberg, M., and Roux, J. (2020). Poroid Hymenochaetaceae associated with trees showing wood-rot symptoms in the garden route national park of South Africa. Mycologia 112, 722-741. doi: 10.1080/00275514.2020.1753160

Tchoumi, J. M. T., Coetzee, M. P. A., Vivas, M., Rajchenberg, M., and Roux, J. (2017). Wood-rotting basidiomycetes associated with declining native trees in timber-harvesting compartments of the garden route national park of South Africa. Austral Ecol. 42, 947-963. doi: 10.1111/aec.12524

The Plant List (2020). A Working List of all Plant Species. Available online at: http://www.theplantlist.org/ [Accessed November 13, 2020]

Tura, D., Zmitrovich, I. V., Wasser, S. P., and Nevo, E. (2009). Medicinal species from genera Inonotus and Phellinus (Aphyllophoromycetideae): culturalmorphological peculiarities, growth characteristics, and qualitative enzymatic activity tests. Int. J. Med. Mushrooms 11, 309-328. doi: 10.1615/IntJMedMushr. v11.i3.100

Tura, D., Zmitrovich, I. V., Wasser, S. P., and Nevo, E. (2010). Checklist of hymenomycetes (Aphyllophorales s.l.) and heterobasidiomycetes in Israel. Mycobiology 38:256. doi: 10.4489/MYCO.2010.38.4.256

Urcelay, C., Robledo, G., Heredia, F., Morera, G., and Montaño, F. G. (2012). Hongos de la Madera en el Arbolado Urbano de Córdoba, 1st Edn. Córdoba: Instituto Multidisciplinario de Biología Vegetal.

Valenzuela, R., Raymundo, T., and Cifuentes, J. (2013). El género Inonotus s.l. (Hymenochaetales: Agaricomycetes) en México. Rev. Mex. Biodivers. 84, S70S90. doi: $10.7550 / \mathrm{rmb} .31605$

Venturella, G., Bernicchia, A., and Saitta, A. (2006). Three rare lignicolous fungi from Sicily (S Italy). Acta Mycol. 41, 95-98. doi: 10.5586/am.20 06.013

Venturella, G., and Raimondo, F. M. (2004). I funghi cariogeni delle alberature di parchi, giardini e strade 1-18. Quad. Bot. Amb. Appl. 15, 181-201.

Vergara, J. I. V., Quintanilla, J. F. L., Inchaurtieta, E. B., Víboras, M. L., Pérez, J. D. R., and García, V. J. E. (2016). Contribución al conocimiento de los hongos del jardín botánico canario "viera y clavijo" (Gran Canaria, Islas Canarias). (II). Bot. Macaronésica 29, 15-24.

Villalobos, S., Mengual, M., and Henao-Mejía, L. G. (2017). Uso de los hongos, Podaxis pistillaris, Inonotus rickii y Phellorinia herculeana (Basidiomycetes), por la Etnia Wayuu en la Alta Guajira Colombiana. Etnobiología 15, $64-73$.

Viveros Garciìa, J. C., Figueroa Rodriiguez, K. A., Gallardo Loìpez, F., Garcì̀a Peìrez, E., Ruiz Rosado, O., and Hernaindez Rosas, F. (2018). Sistemas de manejo y comercializacioin de tamarindo (Tamarindus indica L.) en tres 
municipios de Veracruz. Rev. Mex. Cienc. Agríc. 3, 1217-1230. doi: 10.29312/ remexca.v3i6.1373

Wright, J. E., and Alberto, E. (2006). Guia de la Region Pampeana. II. Hongos Sin Laminillas. Buenos Aires: LOLA.

WWF (2015). The "Big Five" of the Cerrado. Available online at: https: //www.wwf.org.br/informacoes/english/?50242/The [Accessed November 13, 2020]

Xavier-Santos, S., Carvalho, C. C., Bonfá, M., Silva, R., Capelari, M., and Gomes, E. (2004). Screening for pectinolytic activity of wood-rotting Basidiomycetes and characterization of the enzymes. Folia Microbiol. 49, 46-52. doi: 10.1007/ BF02931645

Yuan, Y., Bian, L., Wu, F., and Si, J. (2015). Floral composition and ecology of polypores in Hainan Province. Chinese J. Ecol. 34, 1689-1698.
Zheng, J. J., Lin, Q., Liu, W., and He, X. S. (2011). First discovery of Inonotus rickii on Gleditsia sinensis. Mycosystema 30, 128-132.

Conflict of Interest: The authors declare that the research was conducted in the absence of any commercial or financial relationships that could be construed as a potential conflict of interest.

Copyright (c) 2021 Leonardo-Silva, Abdel-Azeem and Xavier-Santos. This is an openaccess article distributed under the terms of the Creative Commons Attribution License (CC BY). The use, distribution or reproduction in other forums is permitted, provided the original author(s) and the copyright owner(s) are credited and that the original publication in this journal is cited, in accordance with accepted academic practice. No use, distribution or reproduction is permitted which does not comply with these terms. 